\title{
HUMAN TUBERCLE BACILLI IN THE MILK OF A VACCINATED COW.
}

\author{
By A. Stanley Griffith, M.D., Groccrs' Research Scholar.
}

From the Field Laboratories, University of Cambridge.

IT is now generally recognised by veterinary experts that the injection of living human tubercle bacilli, for the purpose of producing an immunity to bovine tuberculosis, is inadmissible in the case of the milch cow, on account of the tendency of the bacilli to pass out of the body with the milk.

For this reason vaccination with a living human virus is being restricted by them to the young calf, in the body of which it is considered to be improbable that the bacilli will remain undestroyed until such time as its flesh or its milk becomes food for man.

But recent investigations have demonstrated that human tubercle bacilli which have been inoculated persist in the body of the bovine animal much longer than was at one time considered likely.

Weber and Titze $\left(1910^{\circ}\right)$ have shown in animals immunised with tauruman and bovo-vaccine (prepared from human cultures) that human tubercle bacilli may multiply in certain bovine tissues (such as those of the eye and the joints), set up localised tuberculosis, and persist within the lesions for many months. In three instances tauruman or bovo-vaccine bacilli were cultivated from a tuberculous eye (twice) or a tuberculous joint (twice) two years and four months to two years and six months after the injection of the bacilli.

It has also been shown that human tubercle bacilli, which have been experimentally inoculated either subcutaneously or intravenously, may settle in the udder and produce tuberculosis of the mammary tissue. Weber and Titze $\left(1907^{3}\right)$ have recorded the occurrence of udder tuberculosis in a cow three years and four months old, which had been inoculated with culture of human tubercle bacilli on four separate occasions, the first inoculation being made intravenously when the animal was eight months old, the other three subcutaneously fifteen, sixteen, and eighteen months subsequently. In the "Appendix to the Final Report of the Royal Commission on Tuberculosis" (1911 1 ) I have put on record the case of a cow (No. 61) which developed tuberculosis of the udder eight months after an intravenous inoculation of human tubercle bacilli. The milk of both these cows produced tuberculosis in guinea-pigs.

These two cases of udder tuberculosis deal with well-grown animals which

${ }^{1}$ Received November 19, 1912. 
were experimentally inoculated with large doses and not with animals vaccinated with small doses when they were young calves.

Notwithstanding, the question arises whether or not there is likelihood of human tubercle bacilli, which have been injected into the young calf for the purpose of producing an immunity to bovine tuberculosis, settling in the immature mamma and surviving therein until the period of lactation.

In the course of investigations, in test of this question, I have obtained evidence that this settlement and survival of human tubercle bacilli in the udder of such auimals with consequent infection of the milk does occur, and that the immunisation of calves against bovine tuberculosis is not, as was supposed, free from danger to the community.

Before giving the details of the case, I will briefly summarise the researches which led up to these investigations.

While acting as investigator to the Royal Commission on Tuberculosis I carried out, during the years 1908-9, a series of investigations upon the milk of animals which had been experimentally inoculated, either subcutaneously or intravenously, with cultures of tubercle bacilli. The experiments were made with the view of ascertaining whether tubercle bacilli would pass from the blood directly into the milk.

Both cows and goats were experimented upon, and it was shown by means of guinea-pig inoculation that tubercle bacilli, human or bovine, which had been injected either into the blood-stream or in the subcutaneous tissues, not only made their appearance in the milk within twenty-four hours of their injection, but also continued to be excreted therein for long periods subsequently.

One cow, No 61, referred to above, was kept under observation 529 days after she had received an intravenous injection of $150 \mathrm{mgrms}$. of a culture of human tubercle bacilli. Human tubercle bacilli were demonstrated in the milk drawn twenty-four hours after her inoculation, and on every subsequent occasion that it was tested up to the forty-fourth day, when she was "dried off." About five and a half months after the intravenous inoculation two of the quarters were milked and some pus was obtained from each; this, examined microscopically, was found to contain very numerous tubercle bacilli. Nearly eight months after the intravenous inoculation the cow developed a tuberculous mastitis, and large quantities of muco-purulent fluid collected in the milk cisterns; this fluid contained numerous tubercle bacilli. The mastitis after a time subsided, the udder atrophied, the milk sinuses contracted, and the teat canals of two of the quarters became closed. Tubercle bacilli were present in large numbers in the purnlent material obtained from the udder on every occasion that it was examined up to the day (the 529th) the cow was killed, and these were shown, by cultivation and rabbit inoculation tests, to be of the lsuman type.

I give the results in this cow rather at length to show what may ensue in an udder, the sinuses of which have become infected with human tubercle bacilli. 
In another case (cow 77), tubercle bacilli were being excreted with the milk when the cow was killed 155 days after she had received a subcutaneous inoculation of 100 mgrms. of human culture. The milk of this cow, during the whole period of the experiment, remained unaltered in quality.

The question of the excretion of human tubercle bacilli in the milk of cows experimentally inoculated with these bacilli has also been investigated by Titze $\left(1908^{5}\right)$. Titze inoculated three cows intravenously with culture of human tubercle bacilli, and then tested the milk upon guinea-pigs. In one experiment tubercle bacilli appeared in the milk within twenty-four hours, and were demonstrated still to be present thirty-eight days after the inoculation of the cow, but not subsequently (99th day). In another experiment the excretion of tubercle bacilli began in the third week, and was being continued on the 144th day, when the cow was slaughtered. In the third experiment three injections of culture were given at intervals of about three months. After the first injection (20 mgrms. human tubercle bacilli) the milk did not become infective for guinea-pigs; nor were tubercle bacilli demonstrated in the milk after the second injection ( 10 mgrms. bovine tubercle bacilli); not until the cow had received the third injection (50 mgrms. human tubercle bacilli) were tubercle bacilli excreted in the milk. It is of interest to note that in each of the three experiments quoted above, the bacilli were excreted by one quarter only, whereas in my experiments all four quarters took part in the excretion.

Since protective vaccination is practised, not upon milch cows, but only upon young calves, and as the doses employed in my cases were relatively very large, these observations upon adult animals seemed to possess merely an academic interest. As stated above, it was thought to be improbable that human tubercle bacilli would remain alive in the tissues of an animal so resistant as the calf is to this type of tubercle bacillus and appear in the milk eighteen to twenty-four months after the injection of the animal.

Nevertheless, as it was a matter upon which definite information was desirable, I extended my observations to some heifer calves which had been inoculated subcutaneously to test the virulence of various sorts of culture, in order to ascertain whether or not tubercle bacilli had been excreted into the milk sinuses of the undeveloped mamma. Material for investigation was obtained from the mamma (by injecting saline and recovering the fluid) of eleven heifer calves, and tubercle bacilli were demonstrated by culture and guinea-pig inoculation to be present in the fluids from no fewer than seven of the animals. In five of the cases they were detected in such fluid microscopically, and in four they were present in such numbers as to suggest that they had multiplied. The calves which gave positive results were 6 to 10 months old when the material was taken and had been under observation 91 to 127 days. Two of the calves had been inoculated with human tubercle bacilli, the others with bovine tubercle bacilli of relatively low bovine virulence (lupus cultures). None of the animals, when killed, showed macroscopic tuberculous lesions of the mammary gland tissue. 
As the work of the Commission was then drawing to its close, I was unable to carry my investigations further, and it remained undetermined whether or not tubercle bacilli, which had got into the mammary sinuses of a calf, would remain alive therein until the mamma became functional.

The persistence, however, with which tubercle bacilli had inhabited the udder of cow 61 raised a strong presumption that the milk of some of these animals, had they been allowed to live and calve, would have contained living tubercle bacilli, and I urged the importance of testing the milk of all cows which had been vaccinated as calves before permitting it to be used as human food.

The opportunity of testing the milk of two heifers vaccinated as calves has recently presented itself to me, and in the milk of one of them I have demonstrated human tubercle bacilli.

This heifer was vaccinated when she was four days old, presumably with a minimal dose, the injection being made intravenously. She calved when she was two years and four months old, and some of the first milk from each of the four quarter's was obtained for examination.

Five guinea-pigs were inoculated with the milk, two with milk from the right fore-quartel and one with milk from each of the other quarters. Three of the guinea-pigs died, one a few hours, the others two and three days after inoculation. Of the two that were left, one inoculated with milk from the right hind-quarter died of general tuberculosis thirty-four days after inoculation; the other, inoculated with milk from the right fore-quarter, killed after the same interval of time, showed early generalised tuberculosis.

From each of the two guinea-pigs which became tuberculous a culture of human type was isolated.

The milk was tested again forty days after the heifer had calved. Four guinea-pigs were inoculated, one from each quarter. Two of the guinea-pigs, inoculated with milk from the hind-quarters, became tuberculous, and a culture from one of them has exhibited the cultural characters of the human tubercle bacillus.

None of the milk of this heifer had been used for human consumption, and when it was known that the second test had given positive results the animal was ordered to be destroyed.

A complete post-mortem examination was not possible, as the animal was killed in the open field. Nothing of an obviously tuberculous nature was found in the udder or in the supramammary lymphatic glands; the mucous membrane of the milk sinuses and ducts was normal, and the milk within them was also normal in appearance. A minute grey tubercle was seen in the lungs, but otherwise they appeared perfectly normal. The thoracic lymphatic glands were normal, and the liver and the spleen and the serous membranes showed no sign of tuberculosis. 
The heifer had reacted to the tuberculin test on three separate occasions, namely, seven, twelve, and twenty-eight months after the protective inoculation.

Milk and emulsions of various tissues of the heifer have been inoculated into guinea-pigs. The results of these will be reported later.

In the meantime I am making this preliminary communication in order to draw attention to the possible danger of using the milk of cows which have been vaccinated, when calves, with human tubercle bacilli.

There is strong probability that the case recorded above is not the only one which has occurred among the vaccinated animals in the same herd. Some months previous to the investigation of the milk of the heifer a (non-vaccinated) calf from the herd was killed because it had reacted to tuberculin; it was examined by Dr. Cobbett and myself and a localised tuberculosis of one mesenteric gland was found; a culture isolated from the gland was of the human type of tubercle bacillus.

Similar consequences do not appear to attend the use of tauruman or bovo-vaccine. Weber and Titze $\left(1910^{4}\right)$ have tested the milk of 79 heifers vaccinated, when calves, with bovo-vaccine and the milk of 8 heifers vaccinated with tauruman without obtaining a single positive result.

But seeing that these observers have isolated human tubercle bacilli from the eyes and joints of animals, two years and four months to two years and six months after they had been injected with these vaccines, it seems to me not unlikely that a case will arise in which it is found that the bacilli have persisted in the mammary tissues.

Whether rare or not, it will be readily appreciated, from what has gone before, how great is the importance of detecting such cases when they do arise.

A cow which is discharging human tubercle bacilli in her milk is a more insidious danger to human health than a cow suffering from bovine tuberculosis. In the latter case, udder tuberculosis does not usually manifest itself until late in the disease. when the animal has for some time perhaps shown clinical evidence of tuberculosis, whereas in the former the milk from the very beginning of lactation contains tubercle bacilli, and may not for many months, though continuing to contain tubercle bacilli in increasing numbers, show any change in quality.

Whatever value the injection of human tubercle bacilli may have in producing immunity in cattle to bovine tuberculosis, it is obvious that the method should not be practised upon animals destined for dairy purposes, in view of this risk of cows becoming carriers of human tubercle bacilli. 
328 HUMAN T. B. IN THE MILK OF A VACCINATED COW.

\section{REFERENCES.}

1. Griffith, A. Stanley . "Appendix to Final Report of the Royal Commission on Tuberculosis," 1911, vol. iii. pp. 83-86.

2. Weber and Trize . . "Tuberculose Arbeiten," 1910, Heft x. S. 191.

3. " " . . . Ibid., 1907, Heft vii. S. 9.

4. " " . . . Ilid., 1910, Heft x. SS. 171 and 183.

5. Tiтze . . . . . Ibid., 1908, Heft ix. S. 50. 